\title{
CARACTERIZACIÓN DE LA CALIDAD DEL SEMEN EN HOMBRES ATENDIDOS EN UN CENTRO DE REPRODUCCIÓN ASISTIDA EN GUAYAQUIL, ECUADOR
}

\author{
Gabriel Morey-León (1) 1,a, Tatiana Puga-Torres (10) 2,a, Xavier Blum-Rojas (102,b, \\ Manuel González-González (iD) 1,c, Alexandra Narváez-Sarasti (iD3,d, Nancy Sorroza-Rojas (iD)3,e \\ 1 Facultad de Ciencias Médicas, Universidad de Guayaquil, Guayaquil, Ecuador. \\ ${ }^{2}$ Centro Nacional de Reproducción Asistida Innaifest, Hospital Clínica Kennedy, Guayaquil, Ecuador. \\ 3 Facultad de Ciencias Médicas, Universidad Espíritu Santo, Samborondón, Ecuador. \\ ${ }^{a}$ Magíster en biotecnología; ${ }^{b}$ doctor en patología clínica; ${ }^{\mathrm{c}}$ magister en virología; ${ }^{\mathrm{d}}$ bióloga; ${ }^{\mathrm{e}}$ magister en bioquímica clínica
}

\section{RESUMEN}

Con el objetivo de caracterizar la calidad seminal de hombres en un centro de reproducción asistida de la ciudad Guayaquil (Ecuador), se colectaron 204 muestras de semen de pacientes con problemas de fertilidad de entre 20 y 57 años, atendidos entre mayo de 2017 y septiembre de 2018. Se realizó un espermograma básico a cada muestra, siguiendo las recomendaciones del manual para la examinación y procesamiento de semen humano. El 27,4\% de las muestras presentó normozoospermia. Dentro de las alteraciones la teratozoospermia fue de $27,9 \%$, oligoteratozoospermia del $8,8 \%$, evidenciándose mayor número en pacientes de 30 a 39 años. Un alto porcentaje de pacientes presentan una calidad del semen y morfología espermática por debajo los limites de referencia establecidos por la Organización Mundial de la Salud.

Palabras clave: Espermatozoides; Infertilidad masculina; Espermograma; Morfología espermática; Teratozoospermia (fuente: DeCS BIREME).

\section{DESCRIPTION OF THE SEMEN QUALITY FROM MEN TREATED IN AN ASSISTED REPRODUCTION CENTER IN GUAYAQUIL, ECUADOR}

\begin{abstract}
In order to characterize the quality of semen from men in an assisted reproduction center in the city of Guayaquil (Ecuador), 204 semen samples were collected from patients with fertility disorders aged 20 to 57 years, who were admitted between May 2017 and September 2018. A basic spermogram was performed on each sample, following the fabricant recommendations for the examination and processing of human semen. It was found that $27.4 \%$ of the samples presented normozoospermia. Among the disorders, it was found that $27.9 \%$ had teratozoospermia, $8.8 \%$ had oligoteratozoospermia and a higher number of patients were found to be between 30 and 39 years old. A high percentage of patients presented sperm morphology and quality values below the reference limits established by the World Health Organization.
\end{abstract}

Keywords: Spermatozoa; Male infertility; Spermogram; Sperm morphology; Teratozoospermia (source: MeSH NLM).

Citar como: Morey-León G, X, González-González M NarváezSarasti A, Sorroza-Rojas N. Caracterización de la calidad del semen en hombres atendidos en un centro de reproducción asistida en Guayaquil, Ecuador. Rev Peru Med Exp Salud Publica. 2020;37(2):2926. doi: https://doi.org/10.17843/ rpmesp.2020.372.4973

Correspondencia: Gabriel MoreyLeón, gabriel.moreyl@ug.edu.ec

Recibido: $17 / 11 / 2019$ Aprobado: 15/04/2020 En línea: 16/06/2020

\section{INTRODUCCIÓN}

La infertilidad es la incapacidad de lograr un embarazo clínico después de 12 meses de relaciones sexuales no protegidas ${ }^{(1-3)}$, afecta del 8 al $15 \%$ de las parejas en todo el mundo ${ }^{(4,5)}$, siendo atribuible al factor masculino en el $50 \%$ de los casos ${ }^{(6,7)}$. Dentro de los factores involucrados en la infertilidad masculina se encuentran los genéticos, el estilo de vida, entre otros ${ }^{(8)}$; sin embargo, cerca del $30 \%$ de los casos aún son considerados como idiopáticos ${ }^{(9)}$.

El análisis de semen es la primera prueba prescrita para el diagnóstico de la fertilidad masculina, esta prueba estudia las características macroscópicas y microscópicas del semen. Proporciona información esencial sobre los parámetros convencionales del espermatozoide, la concentración de espermatozoides y la movilidad, viabilidad y morfología espermática ${ }^{(10)}$. 
El parámetro de la morfología espermática permite identificar el porcentaje de espermatozoides con alteraciones morfológicas ${ }^{(10-14)}$.

El presente trabajo tiene por objetivo caracterizar las principales variaciones en la calidad del semen, incluida las anormalidades en la morfología espermática que se presentan en hombres que acuden a un centro de reproducción asistida de la ciudad Guayaquil (Ecuador).

\section{EL ESTUDIO}

El estudio se realizó en pacientes atendidos en consulta de fertilidad en un centro de reproducción asistida de Guayaquil, entre mayo del 2017 y septiembre del 2018. Se incluyeron hombres de entre 20 y 57 años de edad que iban a realizarse una evaluación de su fertilidad mediante un espermograma básico y que aceptaron participar mediante un consentimiento informado. Se excluyeron los pacientes con antecedentes de enfermedades genéticas y oncológicas que afecten a la fertilidad masculina.

Para la recolección de muestra de semen, los pacientes siguieron los estándares establecidos en el manual de laboratorio para la examinación y procesamiento de semen humano de la OMS ${ }^{(10)}$. Luego de la recolección de la muestra, los pacientes llenaron un formulario con la información de edad, días de abstinencia y fecha de última eyaculación.

El espermograma básico se realizó siguiendo el protocolo establecido en el manual para la examinación y procesamiento de semen humano ${ }^{(10)}$, siendo las muestras de semen evaluadas dentro de la hora de su recolección.

Se evaluaron los parámetros espermáticos de concentración espermática $\left(10^{6}\right.$ por mililitro), la movilidad total (progresiva y no progresiva) y la movilidad progresiva (\%) con una cámara Makler (cámara de recuento Makler; Sefi Medical Instruments, Ltd.) para microscopio invertido. La valoración de la morfología espermática (\%) se realizó mediante la técnica de tinción diferencial de espermatozoides (diff-quik rapid staining con el kit Stat III $^{\circledR}$ Andrology Stain (ref: 85316-I; Mid-Atlantic Diagnostic, Inc.)), siguiendo su protocolo establecido. Los defectos morfológicos fueron clasificados según los criterios estrictos de Kruger del manual

\section{MENSAJES CLAVE}

Motivación para realizar el estudio: La calidad del semen es un parámetro importante en la evaluación de la fertilidad dentro de la pareja con problemas para concebir; sin embargo, no se informa respecto a este problema de salud en los registros nacional y censos del Ecuador (2017).

Principales hallazgos: El presente trabajo identificó en un grupo de pacientes con problemas de fertilidad en Guayaquil (Ecuador) que el 27,4\% de varones tenían los parámetros de calidad del semen debajo de los límites de referencia de la OMS.

Implicancias: El presente estudio permite dar a conocer la importancia de evaluar la fertilidad masculina en la población ecuatoriana, principalmente en parejas con problemas reproductivos.

de la OMS, considerando el área afectada del espermatozoide (cabeza, cuello y cola).

La información fue analizada con el programa InfoStat versión 2019. Se usaron frecuencias y porcentajes para las variables categóricas y la media \pm desviación estándar (DE), para las variables cuantitativas.

El estudio fue aprobado por el Comité de Ética de Investigación en Seres Humanos del Hospital Clínica Kennedy, de Guayaquil (código F.GHK.DOC-04.XI/15). Se respetó la confidencialidad de la información de los pacientes de acuerdo con los principios éticos de la Declaración de Helsinki.

\section{HALLAZGOS}

Se analizaron muestras de semen, mediante espermograma básico, de 204 pacientes, de los cuales, en 76 se evaluó la morfología espermática. La edad media de los pacientes evaluados fue 36,0 \pm 7,1 años. La media de los parámetros analizados y número de casos que no alcanzaron los límites inferiores de referencia establecidos por la quinta edición del manual de la OMS, se describen en la Tabla 1.

Tabla 1. Valores medios de las características seminales cuantitativas de los pacientes

\begin{tabular}{|c|c|c|c|c|}
\hline \multirow{2}{*}{ Característica del semen } & \multirow{2}{*}{ Media \pm DE } & \multirow{2}{*}{$\begin{array}{c}\text { Límites inferiores } \\
\text { de referencia }\end{array}$} & \multicolumn{2}{|c|}{$\begin{array}{l}\text { Individuos con rangos inferiores a los } \\
\text { mínimos de referencia }\end{array}$} \\
\hline & & & Casos & Porcentaje \\
\hline Volumen $(\mathrm{mL})$ & $3,1 \pm 1,4$ & 1,5 & 24 & 10,8 \\
\hline Concentración espermática $\left(10^{6} / \mathrm{mL}\right)$ & $33,8 \pm 31,3$ & 15 & 76 & 37,3 \\
\hline Concentración total (millones) & $103,2 \pm 100,1$ & 39 & 56 & 27,5 \\
\hline Movilidad (\%) & $46,1 \pm 20,4$ & 40 & 66 & 32,4 \\
\hline Total de espermatozoides móviles (millones) & $53,0 \pm 63,2$ & No aplica & No aplica & No aplica \\
\hline Espermatozoides con movilidad progresiva (\%) & $86,2 \pm 18,2$ & 32 & 48 & 23,5 \\
\hline Morfología normal de espermatozoides (\%) & $12,5 \pm 8,1$ & 4 & 41 & 23,3 \\
\hline
\end{tabular}

*Límites de referencia establecidos en el manual de examinación y procesamiento de semen humano de la OMS quinta edición. DE: desviación estándar. 
El porcentaje de pacientes con parámetros de la calidad del semen dentro de los límites inferiores de referencia fue de 27,4\%, mientras que el grupo de pacientes con teratozoospermia fue el mayor grupo de pacientes con la calidad del semen alterado. La Tabla 2 describe la frecuencia de pacientes con diferentes anomalías en la calidad del semen, en base a la nomenclatura establecida en la quinta edición del manual de la OMS.

Dentro del grupo de pacientes con alteraciones en la calidad del semen según la edad del paciente, el grupo de varones de entre 30 y 39 años, presentó una mayor frecuencia de alteraciones en la calidad del semen. La Figura 1 describe el número de pacientes con las alteraciones de la calidad del semen según la edad.

De los pacientes a quienes se les evaluó la morfología espermática, el 23,3\% no se encontró dentro de los límites inferiores de referencia, en estos casos se encontraron mayores defectos en la porción de la cabeza de los espermatozoides. En la Tabla 3 se describe el número de pacientes a quienes se les evaluó la morfología espermática, estratificada por grupos según el porcentaje de normalidad espermática.

\section{DISCUSIÓN}

Dentro del grupo de pacientes evaluados, las medias de todos los parámetros espermáticos analizados estuvieron dentro de los límites de referencia establecidos por la guía de la OMS para la evaluación espermática. Sin embargo, el 37,3\% de los casos no estuvieron dentro de los límites de referencia en uno de sus parámetros estudiados. La frecuencia de teratozoospermia fue la más alta $(27,9 \%)$ dentro de las alteraciones encontradas, seguida por la oligoteratozoospermia $(8,8 \%)$. Se ha comprobado la tendencia decreciente en la calidad del semen en hombres jóvenes fértiles e infértiles ${ }^{(15,16)}$, por ello la OMS, en su manual de análisis de semen humano, ha ido modificando los límites inferiores de referencia de los parámetros seminales, disminuyendo sus valores desde la primera edición hasta la última publicada en el $2010^{(10)}$.

Tabla 2. Clasificación de anormalidades en la calidad del semen de los pacientes.

\begin{tabular}{lcc}
\hline $\begin{array}{l}\text { Nomenclatura relacionada a la } \\
\text { calidad del semen }\end{array}$ & Frecuencia & Porcentaje \\
\hline Teratozoospérmico & 57 & 27,9 \\
Normozoospérmico & 56 & 27,4 \\
Oligoteratozoospérmico & 18 & 8,8 \\
Astenoteratozoospérmico & 16 & 7,8 \\
Oligoastenoteratozoospérmico & 16 & 7,8 \\
Oligozoospérmico & 14 & 6,8 \\
Oligoastenozoospérmico & 9 & 4,4 \\
Azoospérmico & 7 & 3,4 \\
Astenozoospérmico & 6 & 2,9 \\
Criptozoospérmico & 4 & 1,9 \\
Necrozoospérmico & 1 & 0,4 \\
\hline
\end{tabular}

* Clasificación basada en la nomenclatura de la quinta edición del manual de la OMS
Existen trabajos publicados de varios países en los cuales se evalúan los parámetros espermáticos en las diferentes poblaciones masculinas, lo que permite tener una referencia de la calidad seminal de la población específica de ciertos países. No se han encontrado trabajos publicados para la población ecuatoriana donde se caracterice la calidad seminal de los hombres fértiles o infértiles en edad reproductiva. Por ello, el presente trabajo quiso evaluar y caracterizar los parámetros seminales de un grupo de hombres con problemas de fertilidad de la población del Ecuador, para poder tener una referencia de la calidad del semen de esta población.

De todos los pacientes evaluados con el espermograma básico, solo a 176 se les pudo realizar evaluación morfológica de los espermatozoides. El grupo de pacientes al que no se le realizó esta valoración presentó anomalías, que no permitieron obtener un número suficiente de espermatozoides para hacer la tinción correspondiente, como la azoospermia (ausencia total de espermatozoides), criptozoospermia u oligozoospermia severa $\left(<2 \times 10^{6} / \mathrm{mL}\right)$.

Dentro de los parámetros de morfología evaluados se consideraron los defectos de cabeza, cuello y cola de los espermatozoides, y se encontraron más defectos de cabeza que de cuello y cola. Se ha evidenciado que las anormalidades morfológicas de los espermatozoides, especialmente las anomalías en la cabeza espermática, juegan un papel importante en los tratamientos de fertilización in vitro, lo cual afecta más los resultados del tratamiento cuando estas anormalidades morfológicas se encuentran combinadas con otros problemas, como sería el caso de la teratozoospermia severa con oligozoospermia. En este trabajo no se evaluó si los pacientes se sometieron o no a una fertilización in vitro, por lo que no se puede conocer si la morfología espermática influyó en el resultado final del tratamiento.

Se ha descrito la evaluación detallada de incidencia de anormalidades morfológicas de los espermatozoides, como el índice de deformidad espermática (SDI), la teratozoospermia (TZI), o de anomalías múltiples (MAI) ${ }^{(10)}$. Sin embargo, hay poca evidencia en la literatura referente a la relevancia de esta evaluación por alguno de los tres tipos de índices de anormalidades morfológicas descritas ${ }^{(13)}$. En nuestro análisis no se consideró la evaluación de incidencia de anormalidades morfológicas, ya que su uso no forma parte de la rutina diaria en el análisis de la morfología espermática en el laboratorio donde se realizaron los análisis.

Se evidenció mayor número de casos con alteración en la calidad del semen en los pacientes con rango de edad entre los 30 y los 39 años, siendo la teratozoospermia más alta en este rango. Los estudios han evidenciado varios factores que pueden influir en la calidad espermática, como la edad, el estilo de vida, entre otros, los que afectarían principalmente la concentración, la movilidad y la morfología espermática ${ }^{(7,17)}$. Considerando que este grupo etario es la población económicamente más activa en la sociedad, factores como el estrés, 


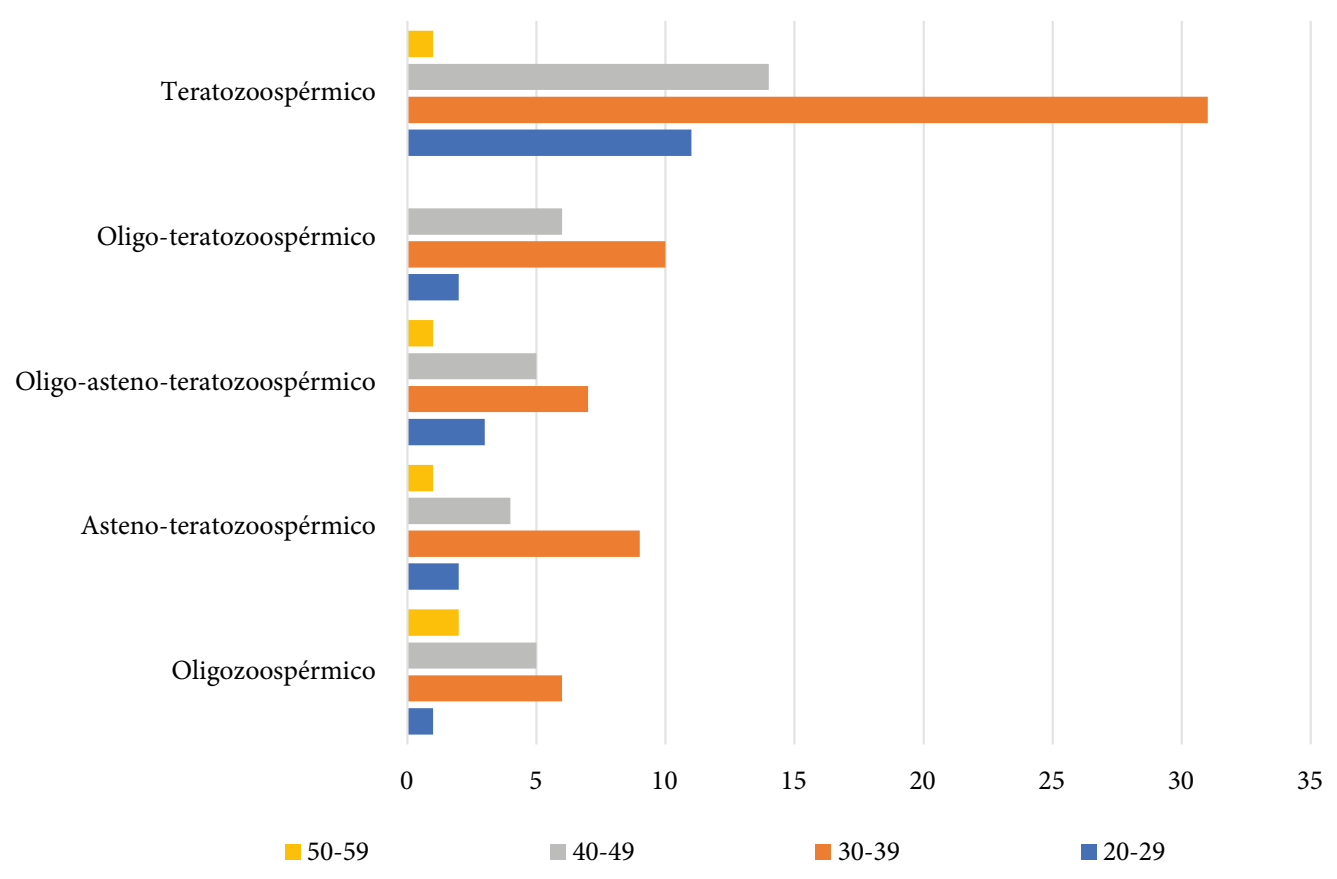

Figura 1. Pacientes con las diferentes alteraciones en la calidad del semen de acuerdo con la edad.

un estilo de vida muy demandante debido a las actividades laborales que pueden involucrar estar mucho tiempo sentado o a los deportes de contacto podrían estar afectando la calidad morfológica de los espermatozoides. Sin embargo, no se evaluaron estos factores en nuestro estudio, por lo que no es posible aseverar que la calidad espermática de esta población se encuentra reducida por algún factor socioambiental. Existen otros factores, como el genético, que pueden influir en la calidad del semen y que afectarían a partes y funciones espermáticas, como la constitución de los flagelos y la movilidad de los espermatozoides ${ }^{(18,19)}$.

En este trabajo solo se incluyeron pacientes que asistieron a un centro de reproducción asistida para evaluar el factor masculino dentro del estudio de la pareja infértil, donde la mala calidad espermática juega un papel importante, por ello no puede ser extrapolado a toda la población masculina infértil de la ciudad y mucho menos del Ecuador.
Los estudios publicados han demostrado diferencias regionales en la calidad del semen, así como una clara disminución de su calidad, por diversos factores ${ }^{(16)}$. Es preocupante el no contar con un registro que permita caracterizar los rangos de calidad espermática en nuestra población y los posibles factores que estén afectando su calidad. La infertilidad por causas del factor masculino podría ser un problema de salud representativo a largo plazo, ya que la población de hombres jóvenes estaría afectando su calidad espermática por factores de estilo de vida moderno y tendrán que recurrir a tratamientos de reproducción para lograr tener descendencia. Por ello, es necesario desarrollar estudios que permitan caracterizar la calidad del semen de la población fértil masculina del Ecuador e identificar las causas que afectan a su calidad a fin de prevenir problemas en la salud reproductiva a futuro.

En conclusión, el presente trabajo ha permitido identificar que los parámetros de la calidad del semen se encuentran por

Tabla 3. Distribución de la concentración, movilidad y defectos espermáticos en relación con la morfología.

\begin{tabular}{|c|c|c|c|c|c|c|c|}
\hline \multirow{2}{*}{$\begin{array}{l}\text { Morfología } \\
\text { espermática } \\
\text { normal }\end{array}$} & \multirow{2}{*}{$\begin{array}{l}\text { Número de } \\
\text { pacientes }\end{array}$} & \multirow{2}{*}{$\begin{array}{c}\text { Concentración } \\
\text { espermática en } \\
10^{6} / \mathrm{mL} \\
(\text { Media } \pm \mathrm{DE})\end{array}$} & \multicolumn{2}{|c|}{$\begin{array}{l}\text { Movilidad en porcentaje } \\
\quad(\text { Media } \pm \text { DE })\end{array}$} & \multicolumn{3}{|c|}{ Defectos espermáticos en porcentaje (Media $\pm \mathrm{DE})$} \\
\hline & & & Total & Progresiva & Cabeza & Cuello & Cola \\
\hline$\leq 4 \%$ & 41 & $23,7 \pm 24,9$ & $25,4 \pm 30,6$ & $81,6 \pm 20,2$ & $50,9 \pm 12,7$ & $24,8 \pm 8,1$ & $21,3 \pm 9,1$ \\
\hline $5-9 \%$ & 25 & $28,6 \pm 21,9$ & $40,0 \pm 43,3$ & $83,0 \pm 22,9$ & $50,6 \pm 9,3$ & $21,7 \pm 6,1$ & $19,8 \pm 8,5$ \\
\hline $10-14 \%$ & 39 & $35,9 \pm 29,3$ & $73,5 \pm 77,1$ & $89,0 \pm 11,2$ & $45,5 \pm 11,1$ & $23,7 \pm 6,6$ & $18,8 \pm 8,3$ \\
\hline$\geq 14 \%$ & 71 & $33,8 \pm 31,2$ & $53,0 \pm 63,4$ & $86,2 \pm 18,6$ & $45,2 \pm 11,4$ & $22,5 \pm 7,0$ & $19,3 \pm 8,4$ \\
\hline
\end{tabular}

DE: desviación estándar 
debajo de los límites de referencia establecidos por la OMS; sin embargo, no es posible extrapolar estos resultados a la población infértil de todo el país. Es necesario realizar un estudio más amplio que permita caracterizar la calidad del semen de la población fértil e infértil de la población ecuatoriana.

Contribución de los autores: GML participó con la asesoría estadística, el análisis e interpretación de datos y la redacción del artículo. TPT contribuyó con la recolección de resultados, la revisión crítica del artículo y la aprobación de la versión final. XBR

\section{REFERENCIAS BIBLIOGRÁFICAS}

1. Zegers-Hochschild F, Adamson GD, de Mouzon J, Ishihara O, Mansour R, Nygren K. on behalf of ICMART and WHO. Glosario de terminología en Técnicas de Reproducción Asistida (TRA). Versión revisada y preparada por el International Committee for Monitoring Assisted Reproductive Technology (ICMART) y la Organización Mundial de la Salud (OMS). OMS 2009. JBRA Assist Reprod 2010; 4(2):19-23.

2. Zegers-Hochschild F, Adamson GD, Dyer S, Racowsky C, de Mouzon J, Sokol R, et al. The international glossary on infertility and fertility care, 2017. Fertil Steril. 2017;108(3):393-406. doi: 10.1016/j.fertnstert.2017.06.005.

3. Vander Borght M, Wyns C. Fertility and infertility: Definition and epidemiology. Clin Biochem. 2018;62:2-10. doi: 10.1016/j.clinbiochem.2018.03.012.

4. Cooper TG, Noonan E, Von Eckardstein S, Auger J, Baker HW, Behre $\mathrm{HM}$, et al. World Health Organization reference values for human semen characteristics. Hum Reprod Update. 2010;16(3): 231-45. doi: 10.1093/ humupd/dmp048.

5. Brugo-Olmedo S, Chillik C, Kopelman S. Definición y causas de la infertilidad. Rev Colomb Obstet Ginecol. 2003;54(4): 227-48.

6. Inhorn MC, Patrizio P. Infertility around the globe: new thinking on gender, reproductive technologies and global movements in the 21st century. Hum Reprod Update. 2015;21(4): 41126. doi: 10.1093/humupd/dmv016.

7. U.S. Department of Health and Human Services, Centers for Disease Control and Prevention, Reproductive Health, Infertility FAQs. 2017. Fecha de acceso: 6 de mayor 2020. Disponible en: https://www.cdc.gov/ reproductivehealth/infertility/.

8. Shi X, Chan CPS, Waters T, Chi L, Chan DYL, Li TC. Lifestyle and demographic factors associated with human semen quality and sperm function. Syst Biol Reprod Med. 2018; 64(5): 358-67. doi: 10.1080/19396368.2018.1491074.

9. Bracke A, Peeters K, Punjabi U, Hoogewijs D, Dewilde SA. Search for molecular mechanisms underlying male idiopathic infertility. Reprod participó con la concepción y el diseño del artículo, el aporte de pacientes o material de estudio y la obtención de financiamiento. MGG participó con la asesoría estadística y asesoría técnica o administrativa. ANS y NSR contribuyeron con el aporte de pacientes o material de estudio, obtención de financiamiento y asesoría técnica o administrativa.

Fuentes de financiamiento: La Universidad Espíritu Santo y el Centro de Reproducción Asistida Innaifest financiaron este trabajo.

Conflictos de interés: Los autores declaran no tener conflictos de intereses

Biomed Online. 2018; 36(3): 327-39. doi: 10.1016/j.rbmo.2017.12.005.

10. World Health Organization. WHOlaboratory manual for the examination and processing of human semen. 2010.

11. Chang V, Garcia A, Hitschfeld N, Härtel S. Gold-standard for computer-assisted morphological sperm analysis. Comput Biol Med. 2017;83: 143-50. doi: 10.1016/j.compbiomed.2017.03.004.

12. Gatimel N, Moreau J, Parinaud J, Léandri RD. Sperm morphology: assessment, pathophysiology, clinical relevance, and state of the art in 2017. Andrology. 2017;5(5): 845-62. doi: 10.1111/andr.12389.

13. Kovac JR, Smith RP, Cajipe M, Lamb DJ, Lipshultz LI. Men with a complete absence of normal sperm morphology exhibit high rates of success without assisted reproduction. Asian J Androl. 2017; 19(1):39-42. doi: 10.4103/1008-682X.189211

14. Instituto Nacional de Estadísticas y Censos (INEC). Recurso Estadístico de Recursos y Actividades de Salud. 2017. Fecha de acceso: 10 de febrero de 2020. Disponible en: https://www.ecuadorencifras.gob.ec/actividades-y-recursos-de-salud/.

15. Virtanen HE, Jørgensen N, Toppari J. Semen quality in the 21 st century. Nat Rev Urol. 2017; 14(2):120.

16. Eisenberg ML, Meldrum D. Effects of age on fertility and sexual function. Fertil Steril. 2017;107(2): 301-04. doi: 10.1016/j.fertnstert.2016.12.018.

17. Ferlin A, Dipresa S, Foresta C. Genetic Testing in Male Infertility. In Human Reproductive and Prenatal Genetics. Academic Press; 2019. p. 383-98.

18. Liu C, Lv M, He X, Zhu Y, Amiri-Yekta A, Li W, et al. Homozygous mutations in SPEF2 induce multiple morphological abnormalities of the sperm flagella and male infertility. J Med Genet. 2019;57(1):31-37. doi: 10.1136/jmedgenet-2019-106011.

19. Coutton C, Martinez G, Kherraf ZE, Amiri-Yekta A, Boguenet M, Saut $\mathrm{A}$, et al. Bi-allelic mutations in ARMC2 lead to severe Astheno-Teratozoospermia due to sperm flagellum malformations in humans and mice. Am J Hum Genet. 2019; 104(2):331-40. doi: 10.1016/j.ajhg.2018.12.013. 